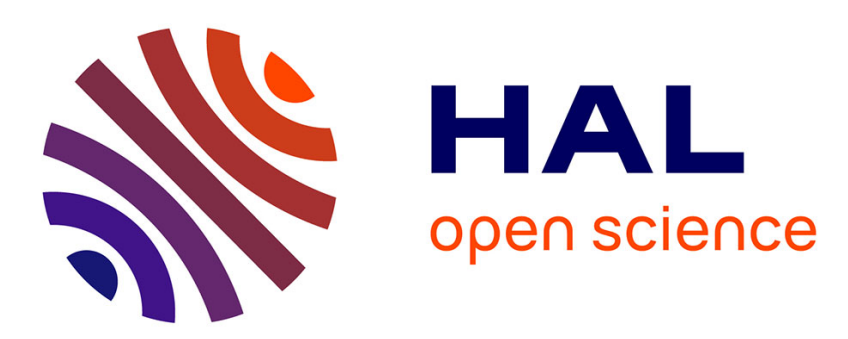

\title{
Réguler les universités par les instruments. Expériences de l'enseignement supérieur au Kenya et en Ouganda
}

Olivier Provini

\section{To cite this version:}

Olivier Provini. Réguler les universités par les instruments. Expériences de l'enseignement supérieur au Kenya et en Ouganda. Revue internationale de politique comparée, 2020, 27 (2-3), pp.87-110. hal-03181889

\section{HAL Id: hal-03181889 \\ https://hal.science/hal-03181889}

Submitted on 26 Mar 2021

HAL is a multi-disciplinary open access archive for the deposit and dissemination of scientific research documents, whether they are published or not. The documents may come from teaching and research institutions in France or abroad, or from public or private research centers.
L'archive ouverte pluridisciplinaire HAL, est destinée au dépôt et à la diffusion de documents scientifiques de niveau recherche, publiés ou non, émanant des établissements d'enseignement et de recherche français ou étrangers, des laboratoires publics ou privés. 


\title{
Réguler les universités par les instruments. Expériences de l'enseignement supérieur au Kenya et en Ouganda
}

\begin{abstract}
$* * *$
Olivier Provini

Maître de conférences en science politique

Université de La Réunion

olivier.provini@univ-reunion.fr

\section{Résumé}

L'objectif de cet article consiste à discuter - à partir des exemples kenyan et ougandais - les ressorts de la nouvelle ingénierie universitaire qui se fabrique et se met en œuvre dans la région de l'Afrique de l'Est. Deux principaux instruments structurant le secteur de l'enseignement supérieur sont pris en exemple : les normes d'assurance qualité et les objectifs nationaux régulant, dans les programmes de développement des gouvernements, les pratiques sur les savoirs. Nous démontrons que lorsque l'on s'arrête sur la fabrique de ces Instruments d'Action Publique (IAP), nos deux études de cas présentent des trajectoires similaires, tant sur la nature de ces instruments que sur les acteurs qui les impulsent. Pour autant, les cas kenyans et ougandais donnent à voir des résultats contrastés sur la mise en œuvre des IAP et, plus largement, sur les réformes d'enseignement supérieur. Alors que le cas kenyan de l'Université de Nairobi valide l'importance prise par les IAP dans la régulation du secteur et de ses institutions, l'exemple ougandais de l'Université de Makerere illustre plutôt les décalages et les effets originaux et non-désirés qui peuvent se produire lors de l'implémentation des IAP.
\end{abstract}

\section{Mots clés}

Instrument d'action publique ; analyse des politiques publiques ; fabrique ; mise en œuvre ; université ; Kenya ; Ouganda.

\section{Introduction $^{1}$}

A la fin des années 2000, les administrateurs de 1'Université de Nairobi (UN) signent un partenariat avec l'institut national «Kenya Bureau of Standards » ayant pour mission de certifier les activités des institutions publiques comme privées du pays (Provini, 2019). Pour l'UN, il s'agit de mettre en place la norme ISO 9001, norme de management de la qualité issu du secteur privé, afin de réguler l'intégralité des procédures, des formations et des offres existantes. Le 7 août 2008, George A. O. Magoha, Vice-Chancelier de l'UN, affirme à l'occasion de la présentation officielle de l'accord : "As the leading institution of higher

\footnotetext{
${ }^{1}$ Ce papier est issu d'une communication lors du Congrès national de l'Association française de science politique (Montpellier, 2017). Nous tenons d'ailleurs à remercier Lydie Cabane, Charlotte Halpern et Pauline Ravinet pour leurs commentaires sur des versions antérieures de cet article ainsi que les évaluateurs anonymes pour leurs remarques stimulantes.
} 
learning in the country, the University of Nairobi continues to play a critical role in the production of high level manpower needed for national development. This role has in the recent past been threatened by emerging challenges characterized by diminishing resources in the face of increasing demand for higher education. To surmount these challenges, the University embarked on a reform programme whose ultimate goal was to transform the University into a modern institution of higher learning comparable to the best in the world. Part of the reform programme entailed [the] introduction of a Quality Management System that would ensure the institution meets customer requirements as well as statutory and regulatory needs pertinent to University services [...]. In achieving this milestone, the University of Nairobi becomes one of the very few public institutions in the country to attain this international standard of excellence. We commit ourselves to not only maintaining the Quality Management System we have developed, but also ensuring its continued improvement $\|^{2}$. La norme ISO consacre une hiérarchisation des valeurs où la recherche de la performance et de la rentabilité est désormais organisée comme la matrice des activités de l'établissement public. La certification des activités de l'UN s'inscrit, plus largement, dans la volonté du gouvernement de Mwai Kibaki (2002-2013) d'accroître l'encadrement des procédures et des services délivrés dans les institutions publiques ${ }^{3}$. Le secteur de l'enseignement supérieur kenyan constitue un parangon de cette décision politique puisque l'ensemble des universités publiques adopte progressivement la norme ISO 9001, entraînant même une émulation dans la gestion des établissements privés. La consécration de cette nouvelle manière de gouverner par la certification - inédite pour le système universitaire n'est qu'un épisode de la régulation systématisée par des Instruments d'Action Publique (IAP) du secteur de l'enseignement supérieur en Afrique de l'Est. Ce concept d'IAP est, dans la littérature francophone, issu des travaux de P. Lascoumes et $\mathrm{P}$. Le Galès qui définissent un instrument comme une institution au sens sociologique du terme, c'est-à-dire comme un ensemble de procédures et de règles qui encadrent les interactions et les comportements des acteurs. Ainsi, les IAP dans les universités sont des dispositifs techniques et sociaux - par exemple, la norme ISO - qui structurent les rapports sociaux dans les établissements en fonction des significations et des représentations - par exemple, l'avènement d'un nouveau management public - dont ils sont porteurs (Lascoumes, 2004 ; Lascoumes et Le Galès, 2010 ; Lascoumes et Simard, 2011; Halpern et al., 2014).

L'objectif de cet article consiste à discuter - à partir des exemples comparés kenyan et ougandais et de deux instruments déployés dans les établissements - les ressorts de cette nouvelle ingénierie universitaire qui demeure peu travaillée dans la région et sur le continent (Lebeau, 2003; Materu, 2007 ; Uwakwe et al., 2008 ; Provini, 2012 ; Tade, 2012 ; Munene 2013 ; Shabani et al., 2017). Le choix du Kenya et de l'Ouganda s'explique, d'une part, par le rôle moteur des deux pays dans le fonctionnement de la Communauté d'Afrique de l'Est (EAC). Cette dernière est actuellement l'une des organisations régionales, notamment concernant le secteur de l'enseignement supérieur, les plus dynamique du continent (Léon, 2004). D'autre part, leurs deux établissements publics historiques partagent un héritage universitaire et des pratiques académiques anglophones en commun. Ils ont participé à former, entre 1963 et 1970, l'Université d'Afrique de l'Est, collège indépendant de l'Université de Londres (Mngomezulu, 2004; Kithinji, 2012), ce qui a favorisé les collaborations et les échanges institutionnels. Jusqu'à présent la littérature sur les réformes universitaires en Afrique, y compris sur la région, s'est surtout attachée à étudier les

\footnotetext{
2 Discours de George A. O. Magoha, Vice-Chancelier de 1'UN, consulté le 23/09/2011 sur http://archive2.uonbi.ac.ke/iso certification files/speeches/vice chancellor.pdf.

${ }^{3}$ Entretien avec un acteur en charge du secteur social du programme de développement « Vision 2030 », réalisé le 29/10/2012, Nairobi.
} 
conséquences sociales des réformes, notamment afin de mettre en exergue les mécanismes de mise en marché des systèmes universitaires. Ces travaux défendent majoritairement la thèse que les gouvernements et les établissements ont adopté, comme telles, les prescriptions internationales de construction d'un marché de l'enseignement supérieur, discours portés par les institutions financières internationales et relayés par les bailleurs de fonds dans le cadre des conditionnalités de l'aide au développement (voir une synthèse dans Brock-Utne, 2003 ; Zeleza et Olukoshi, 2004 ; Charlier, 2006 ; Charton et Owuor, 2008 ; Akkari et Payet, 2010 ; Charlier et Croché, 2010 ; Leclerc-Olive et al., 2011 ; Bugwabari et al., 2012 ; Charlier et Croché, 2012 ; Lange et Henaff, 2015 ; Knight et Woldegiorgis, 2017). Notre article propose plutôt de déplacer le regard sur une transformation singulière du champ académique kenyan et ougandais : l'implémentation d'une nouvelle ingénierie dans la régulation des institutions d'enseignement supérieur. A partir de cet objet de recherche singulier - les IAP dans l'enseignement supérieur -, plusieurs questions ont guidé notre réflexion : que nous apporte cette entrée par les IAP pour penser les réformes des systèmes universitaires ? Comment ces IAP se matérialisent-ils sur la problématique de l'enseignement supérieur en Afrique de l'Est? Qui sont les acteurs qui participent à la fabrique et la mise en œuvre de ces IAP ? Comment se sont effectuées les greffes au niveau des universités, c'est-à-dire de quelles manières ces nouveaux modes de gouvernement sont-ils (re)négociés et mis en œuvre par les acteurs locaux ? Et, finalement, comment ces transformations peuvent-elles se révéler être contradictoires aux objectifs affichés initialement?

Dans l'article, les IAP au sein des établissements d'enseignement supérieur se déclinent dans deux domaines spécifiques : les politiques de régionalisation de l'assurance qualité promues par la Communauté d'Afrique de l'Est, d'une part, et les ambitieux programmes de développement promus nationalement par les gouvernements, d'autre part. Le choix de retenir ces deux IAP s'explique par le résultat de notre recherche empirique et notamment de nos entretiens semi-directifs. En effet, ils ressortent comme les instruments les plus structurants actuellement du champ universitaire est-africain. A partir de cette comparaison entre les pays et les IAP, nous démontrons que lorsque l'on s'arrête sur la séquence de la fabrique, les deux études de cas présentent des trajectoires similaires et attestent du rôle central des IAP dans la régulation des universités. Pour autant, la mise en œuvre des IAP invite à nuancer ces premiers résultats. En effet, le cas kenyan démontre comment, par la norme ISO, de nouvelles procédures et normes bureaucratiques régulent l'UN et comment s'organisent des manières inédites de mettre en réforme les universités publiques. Alors que le cas kenyan valide l'importance prise par les IAP dans les établissements d'enseignement supérieur, l'exemple ougandais de l'Université de Makerere (UM) illustre plutôt les décalages et les effets originaux et non-désirés lors de l'implémentation des IAP. Le cas ougandais met en exergue comment se greffent de nouveaux acteurs lors de la mise en œuvre et comment les IAP sont négociés localement. Cette approche par les instruments apporte un regard éclairant sur les configurations à l'œuvre et rejoint les résultats classiques de l'analyse des politiques publiques sur le décalage entre la fabrique et l'implémentation de l'action publique.

Cet article est issu d'une recherche comparative sur la circulation des réformes universitaires en Afrique de l'Est (Provini, 2015). L'argumentaire s'appuie sur plusieurs enquêtes de terrains réalisées au Kenya (octobre 2011-janvier 2012; mars 2012 et septembre-octobre 2012 à Nairobi) et en Ouganda (avril-juin 2012 et novembre-décembre 2012 à Kampala). D'un point de vue méthodologique, 103 entretiens semi-directifs et discussions informelles avec plusieurs profils - étudiants, enseignants, membres du personnel administratif, responsables des associations étudiantes et enseignantes, dirigeants politiques, bailleurs de fonds et organismes internationaux - ont été réalisés. De nombreuses données quantitatives 
ont été mobilisées, notamment au sein des administrations centrales, et un ensemble d'articles dans les quotidiens et hebdomadaires anglophones a été récolté, entre le milieu des années 1980 et le début des années 2010, afin d'historiciser les différentes phases et modalités de réformes. Concernant l'organisation de l'argumentaire et la construction de la comparaison, dans la première partie de l'article, nous revenons sur la fabrique des IAP en Afrique de l'Est en s'arrêtant sur le rôle des acteurs régionaux et gouvernementaux. Pour nos deux études de cas dans cette partie, nous nous concentrons sur la fabrique des normes d'assurance qualité et des objectifs nationaux régulant les pratiques sur les savoirs. L'objectif de la seconde partie est de déplacer le regard en s'intéressant à la séquence de la mise en œuvre de ces IAP au sein de deux établissements publics d'enseignement supérieur: le cas de l'UN au Kenya est essentiellement centré sur l'assurance qualité alors que l'étude sur l'UM en Ouganda traite surtout des programmes de développement national et leurs conséquences sur les savoirs. Les données disponibles et récoltées durant les terrains de thèse ne nous permettent pas de faire une comparaison systématique, même si des éléments sur la faiblesse institutionnelle de l'assurance qualité à l'UM sont rappelés. Au delà de la comparaison, l'intérêt de ce regard croisé consiste surtout à inscrire les résultats contrastés sur la mise en œuvre dans les controverses scientifiques sur les IAP - structurantes du dossier - et à l'analyse des politiques publiques. Il est ainsi rappelé, à partir des nos études de cas, combien il est important que les analyses scientifiques mettent en exergue les potentielles dynamiques de blocage, de conflit et d'opposition dans la mise en œuvre des IAP.

\section{La fabrique d'une nouvelle ingénierie universitaire en Afrique de l'Est : le rôle structurant des acteurs régionaux et nationaux}

Dans cette première partie, nous revenons sur la fabrique des IAP en Afrique de l'Est et le rôle des acteurs régionaux et nationaux dans ces processus. Plus précisément, deux principaux instruments structurent le secteur de l'enseignement supérieur au Kenya et en Ouganda : les normes d'assurance qualité promues par la régionalisation des politiques publiques d'enseignement supérieur et les objectifs nationaux régulant, dans les programmes de développement des gouvernements, les pratiques sur les savoirs.

\subsection{Le rôle de la Communauté d'Afrique de l'Est dans la promotion des normes d'assurance qualité}

Depuis le milieu des années 1960, le Kenya et l'Ouganda sont membres de l'EAC qui s'est progressivement organisé comme un marché commun de libre-échange et qui tend à se structurer comme une organisation politique. Au sein de l'EAC, le Conseil interuniversitaire pour l'Afrique de l'Est (IUCEA) coordonne et pilote, avec les différentes commissions nationales de l'enseignement supérieur de chaque pays membre, les politiques universitaires au niveau est-africain. Comme l'explique le secrétaire exécutif de l'IUECA, l'institution tend à harmoniser les politiques de l'enseignement supérieur tout en respectant la souveraineté de ses Etats membres : "Standardisation is making everything the same. And this is not possible. Because higher education is the prerogative of the sovereign State. And in this community [Communauté d'Afrique de l'Est], each of five countries are sovereign States. And they have their own agenda as far as the higher education is concerned. So each country has its own particularities. However, together, we have to harmonize. So the basics have to be the same » ${ }^{4}$. Les principaux objectifs de l'IUCEA consistent, en effet, à faciliter les contacts entre les universités d'Afrique de l'Est, à fournir une arène de discussion aux acteurs de

\footnotetext{
${ }^{4}$ Entretien réalisé le 19/11/2012, Kampala.
} 
l'enseignement supérieur, à harmoniser les systèmes universitaires, les programmes et cursus et, enfin, à mettre en place des standards académiques et de qualité comparables (The InterUniversity Council for East Africa et East African Community, 2009 : 11-15). L'IUCEA s'est progressivement constituée comme une institution qui favorise la circulation d'un savoir-faire technique sur l'enseignement supérieur en diffusant une certaine vision de l'université et en promouvant le développement d'une nouvelle ingénierie universitaire dans la région. En 2006, l'IUCEA, le Service Allemand des Echanges Universitaires (DAAD) et la Conférence des recteurs universitaires allemands signent un partenariat pour promouvoir des programmes d'assurance qualité au sein des universités publiques et privées est-africaines ${ }^{5}$. L'agence de coopération allemande investit 1,4 millions d'euros entre 2006 et 2012. Ce fonds permet aux personnels - administratifs comme enseignants-chercheurs - de quarante-cinq universités de suivre des formations spécialisées sur l'assurance qualité et sur les procédures d'audit en Allemagne entre 2007 et $2008^{6}$, d'organiser des workshops dans la région et de rédiger des documents cadres fixant les benchmarks et standards régionaux de ces nouvelles normes bureaucratiques (The Inter-University Council for East Africa, 2010a, 2010b) : "These quality assurance coordinators were trained on the use of the handbook [The Road Map to Quality : A Handbook for Quality Assurance in Higher Education] and on subjects such as the nature of quality assurance, how to carry out self-assessment and external assessment at program level and how to establish internal quality assurance systems in a higher institution of learning » (Hansert et al., $2012: 7$ ).

Cette « bureaucratisation des procédures au nom de la transparence » (Bayart, 2012:99) est structurée par des espaces communs de socialisation, des techniques et outils de quantification et d'évaluation : mise en place de formation, d'audits internes et externes, établissement de nouvelles normes, rédaction de nombreux manuels, plans et autres programmes de développement aux objectifs chiffrés. Ces prescriptions de l'IUCEA ont eu comme conséquence la mise en place de bureaux d'assurance qualité au sein des universités publiques et privées pour coordonner les procédures d'audits internes et externes. L'objectif est de créer des « gold standards » (Hansert et al., 2012:8) afin d'encadrer les procédures de management bureaucratique, l'accréditation des programmes universitaires et former une nouvelle catégorie de managers universitaires épousant cette nouvelle rationalité. Enfin, l'ensemble de ces outils favorise la comparaison, le classement et la compétitivité entre les établissements d'enseignement supérieur dans la région et sur le continent. Cette consécration de la performance par les IAP se retrouve en filigrane du discours de Joseph B. Wanjui, Chancelier de l'UN, lors de l'inauguration du Conseil de l'UN le 17 août 2011 : « You will be serving on the Council of the largest, oldest, most diverse and prestigious University in the region. This is a premier institution in the country that is looked up to, for leadership in every regard. This perception is corroborated by our outstanding performance in the government performance contracting in which we have remained top performers. In the last two years, we were ranked positions two and three in a field of more than 169 state corporations. It is worth noting that many corporations with stronger financial positions have trailed UN in the last two years which confirms the determination by management to remain competitive despite lack of enough resources. Even internationally, we are ranked top locally and among the leading universities in the region. Your duty and responsibility therefore is to ensure that the

\footnotetext{
${ }^{5}$ Entretien avec un responsable du bureau Afrique du DAAD, réalisé le 28/01/2013 à Nairobi.

${ }^{6}$ Entretien avec l'un des coordinateurs du projet de l'assurance qualité en Afrique de l'Est, réalisé le 18 octobre 2012 à Nairobi et plusieurs discussions informelles lors de la conférence de l'IUCEA à Arusha (Tanzanie) les 22-26 octobre 2012 .
} 
University grows to become a strong, robust and excellent institution ${ }^{7}$. Ce modèle d'assurance qualité pour les universités de la région - financées par la coopération allemande - est imaginé à partir des expériences des établissements européens (Hansert et al., 2012 : 9). L'objet de l'assurance qualité est un nouvel exemple saillant pour le continent africain de la circulation des politiques publiques d'enseignement supérieur (Provini 2015).

\subsection{Le rôle des programmes de développement national au Kenya et en Ouganda dans la régulation des savoirs du secteur de l'enseignement supérieur}

Au-delà des politiques promouvant les normes d'assurance qualité, le secteur de l'enseignement supérieur en Ouganda et au Kenya est également agencé par des instruments ayant des effets directs sur la recomposition des cursus et des savoirs : ce sont les projets de développement national (visions 2025, 2030, 2035 et 2040). Ces derniers engagent de nouvelles réflexions sur les formations et les cursus que doivent proposer les universités (Government of the Republic of Kenya, 2007, 2012 ; The Republic of Uganda, 1998, 2006, 2013). Ces projets s'inscrivent dans les politiques de développement et de planification que connaissent actuellement - de nouveau - de nombreux Etats africains (Samuel, 2017 ; Speich, 2017) et qui traduisent l'avènement d'une ingénierie planificatrice réinventée et des modes originaux de gouvernement. Ces programmes de développement national sont des plans de réformes à moyen terme lancé le plus souvent sous l'impulsion des institutions internationales et de leurs experts. Par exemple, le programme kenyan de développement « Vision 2030 » a été piloté par des experts internationaux provenant de la Corée du Sud, du Japon, de Singapour, de la Malaisie et de la Chine (Fourie, 2014, p. 545-546). Ce regard vers 1'Asie est une défiance vis-à-vis des institutions internationales et confirme la volonté des élites estafricaines de construire des partenariats Sud-Sud : " au Kenya, il y a actuellement chez les élites un rejet des tentatives d'influence de l'Occident, et plus encore de la Banque mondiale et du FMI. Ils veulent prendre leur autonomie, notamment à travers leur rapport à une Asie qui est en train d'inventer de nouvelles valeurs, de nouvelles visions, de nouvelles façons de travailler ensemble » (entretien d'H. Maupeu dans Coloma, 2013). Ce parangon asiatique prend forme par l'utilisation répétée du « benchmarking 》- ou étalonnage des performances à travers la mise en scène de nombreux graphiques, schémas et tableaux. L'objectif de l'instrument est, à travers l'utilisation d'indicateurs chiffrés, d'identifier un modèle pour se comparer et de mesurer l'écart existant pour mettre en place des stratégies visant à le combler (Bruno, 2008). Ces «visions » sont construites autour d'indicateurs sectoriels (économie, énergie, tourisme, éducation...) et produisent une comparaison internationale, notamment avec la trajectoire des dragons asiatiques - par exemple avec la Malaisie, la Chine, l’Indonésie, la Thaïlande, l'Inde, le Bangladesh et le Vietnam.

Au sein de ces programmes de développement national, le secteur universitaire occupe une place stratégique afin de promouvoir, par l'enseignement supérieur, l'investissement et favoriser le développement des filières scientifiques. Cette stratégie de promotion des sciences et de la technologie se décline dans trois domaines au Kenya et en Ouganda: l'investissement dans le secteur de la recherche, l'équilibre entre les étudiants inscrits en sciences et en sciences sociales et la transformation des curricula délivrés par les universités. En effet, les formations offertes au sein des établissements publics et privés doivent mieux correspondre aux priorités de développement fixés par le gouvernement afin d'améliorer l'employabilité et de répondre plus efficacement aux besoins de main-d'œuvre du marché.

\footnotetext{
7 Consulté sur le site Internet de l'établissement le 23/09/2011. Voir http://www.uonbi.ac.ke/sites/default/files/CHANCELLOR'S SPEECH INAUGURATION OF THE UON C OUNCIL.pdf.
} 
Ces programmes de développement national ont engagé une réflexion sur les savoirs enseignés à l'université, sur leur adaptabilité au marché, aux besoins économiques et à l'offre - par exemple pour la formation des cadres et des managers de l'administration publique. Au niveau des contenus d'enseignement, de nombreuses modifications doivent donc être engagées par les universités pour que les cursus correspondent à ces priorités nationales. Cette nouvelle ingénierie a comme caractéristique de promouvoir les filières scientifiques au détriment des filières en Sciences Humaines et Sociales (SHS). Les programme kenyans « Vision 2030 » et ougandais « Vision 2040 » recommandent, par exemple, de consolider de nouveaux partenariats entre les universités et les entreprises et de renforcer les investissements dans la recherche scientifique et dans les secteurs jugés prioritaires: la science, l'ingénierie, l'énergie, l'agriculture, le commerce, les télécommunications, les technologies ou encore l'informatique (Government of the Republic of Kenya, 2007 ; The Republic of Uganda, 2013). Enfin, l'objectif est de délivrer, prioritairement, des bourses d'étude aux étudiants inscrits dans les filières scientifiques pour les encourager à privilégier ces cursus. Les secteurs de l'enseignement supérieur au Kenya et en Ouganda sont régulés par deux principaux IAP : les normes d'assurance qualité promues par les acteurs régionaux et les programmes de développement national. Nos deux cas d'étude présentent - lorsqu'on s'intéresse à la séquence de la fabrique des IAP - des logiques similaires : la nature des instruments est comparable et les acteurs promouvant leur développement sont analogues.

\section{La mise en œuvre des IAP : les résultats contrastés au Kenya et en Ouganda}

L'objectif de l'article est désormais de s'intéresser à la séquence de la mise en œuvre de ces IAP au sein de deux établissements publics d'enseignement supérieur : l'UN au Kenya et l'UM en Ouganda. En s'arrêtant sur l'implémentation de ces IAP, les deux cas d'étude proposent des expériences contrastées. Le cas kenyan valide l'importance prise par les IAP dans la régulation des établissements d'enseignement supérieur alors que l'UM illustre, plutôt, les décalages et les effets originaux et non-désirés lors de l'implémentation. Plus précisément, le cas de l'UN démontre l'importance prise par les normes d'assurance qualité alors que l'UM est caractérisée par une faiblesse institutionnelle rendant cosmétique la mise en œuvre des IAP. Surtout, l'étude de cas ougandaise sur les programmes de développement national et leurs conséquences sur les savoirs nous rappelle combien les IAP peuvent se superposer à des enjeux locaux.

\subsection{L'avènement d'une gestion managériale de l'université : l'exemple de la mise en œuvre de l'ISO 9001 à l'UN}

La certification ISO 9001 est une norme issue du monde de l'entreprise. Elle définit des exigences et des lignes directrices pour assurer les services et les processus internes à un organisme en traduisant une série d'exigences dans le système de management, quelque soit sa taille ou son secteur d'activité. De nouvelles procédures sont créées autour d'indicateurs de qualité en vue de passer, avec succès, l'audit conduit par un organisme certificateur. L'objectif est, ensuite, de s'efforcer d'améliorer sans cesse le service rendu, puisqu'une fois certifié, l'établissement est contrôlé et la certification renouvelée, sur la base de ces indicateurs de qualité. Avec l'adoption de la norme ISO 9001, l'UN semble s'instaurer comme un véritable prestataire au service de l'économie du savoir (Provini 2019). L'ISO 9001 consacre le changement de paradigme : l'entreprise universitaire se structure désormais par des consommateurs et clients à satisfaire - anciennement étudiants -, des biens consommables - contenus d'enseignement -, des nouvelles exigences face au développement national par l'essor d'une expertise utile et rentable - recherche -, l'ensemble étant encadré 
par des managers - administrateurs - et par une gouvernance moderne et performante administration (Charton, Owuor, 2008). Ainsi, la certification ISO 9001 transforme les pratiques académiques et administratives au sein des établissements en mimant le fonctionnement du marché. Les carrières professionnelles des enseignants et des professeurs de l'UN sont désormais encadrées par des "contrats de performance » qui façonnent les pratiques académiques et les promotions. Un enseignant formule, en ces termes, sa compréhension de ces nouveaux contrats : «On travaille, on est obligé de travailler. On a des contrats à remplir, ça s'appelle des « contrats de performance ». Il faut qu'on enseigne, il faut également publier [...]. Pour chaque année, pour chaque professeur, c'est pour tout le monde. Il faut publier, il faut qu'on montre qu'on travaille sur quelque chose [...]. Il faut également faire des conférences, participer à des colloques régionaux et internationaux [...]. A chaque quart de semestre, on doit faire un compte-rendu. Mais une fois par an, on remplit une sorte de formulaire avec le chef de département et les pairs, c'est-à-dire les collègues. Et ils doivent cocher $»^{8}$. Parmi le corps enseignant, l'ISO est d'ailleurs appréhendé comme un acteur à part entière $\mathrm{du}$ fonctionnement quotidien de l'université. Jouissant d'une personnification étonnante, l'ISO est considéré comme un agent encadrant les pratiques et les représentations des universitaires : " On a des problèmes pour corriger les copies, parce qu'avec l'ISO, on n'a que deux semaines. Il faut rendre les copies deux semaines après les examens. Donc c'est un peu difficile pour nous, mais c'est l'ISO qui dit qu'on doit le faire, on est done obligé de le faire $\gg{ }^{9}$. Véritable marque et label de $1^{\prime} \mathrm{UN}$ qui se conjugue en porte-clés, stylos, portails de messagerie et cartes étudiantes, l'ISO est fièrement arboré sur l'ensemble des campus par des affiches et des encadrés où sont arborés les trois fétiches (Bayart, 2012: 99) de la certification: "vision», "mission» et "core values». Ces derniềres sont, par exemple, "l'inventivité et la créativité », « la bonne gouvernance entrepreneuriale » ou encore "un service clientèle de qualité » (Provini, 2012).

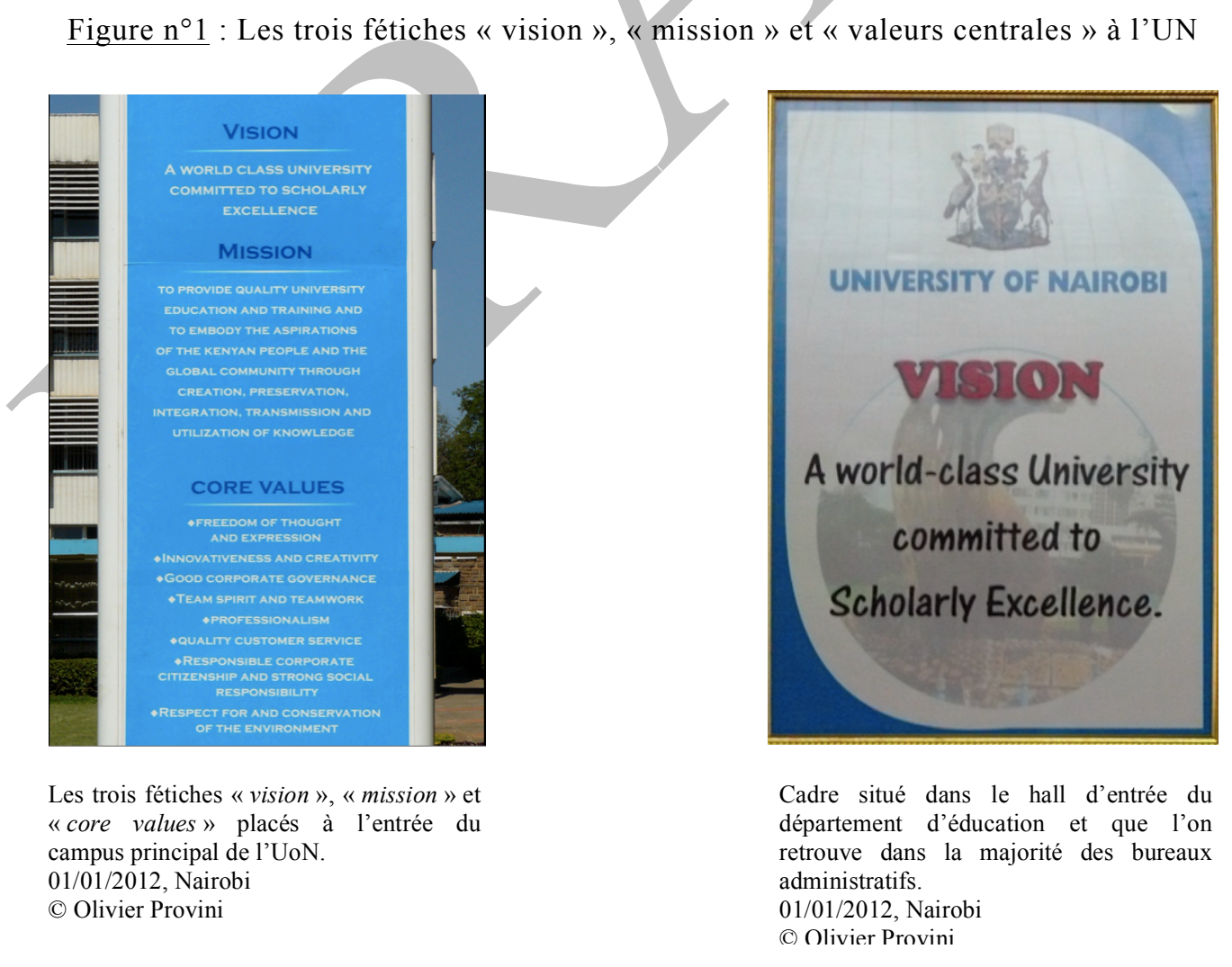

\footnotetext{
${ }^{8}$ Entretien réalisé le 30/11/2011, Nairobi.

${ }^{9}$ Entretien réalisé le 30/11/2011, Nairobi.
} 
L'université a également adopté une charte énumérant les services délivrés. Elle est disposée à l'entrée des départements en versions swahili et anglophone. A chaque service fourni correspond une «condition requise », un " coût » et une "durée d'attente ». On y apprend, par exemple, que le temps d'exécution imparti pour un renseignement téléphonique ne doit pas dépasser vingt secondes. L'ensemble de ces procédés est bien plus qu'une simple mise sur papier des procédures bureaucratiques. Il consacre une étape supplémentaire dans la managérialisation de l'enseignement supérieur où doit s'organiser une discipline des administrateurs et des enseignants devant les intérêts des étudiants-clients. Comme l'expliquent Pierre Dardot et Christian Laval dans leur réflexion sur le gouvernement entrepreneurial, «avec les nouveaux dispositifs de contrôle se développent de nouvelles perceptions des tâches à accomplir, de nouveaux rapports au travail et aux autres [...]. L'intériorisation des normes de performance, l'auto-surveillance constante pour se conformer aux indicateurs, la compétition avec les autres sont les ingrédients de cette révolution des mentalités que les modernisateurs veulent opérer» (Dardot et Laval, 2009 : 387-398). Surtout, cette rationalité a permis le fleurissement de nouveaux enjeux économiques. Autour de la certification des universités publiques, privées et des établissements techniques au Kenya gravite un ensemble d'organismes publics et privés qui se greffe au long processus d'accréditation, que les institutions universitaires doivent renouveler tous les trois ans pour conserver leur certification. Alors que ces procédures annoncent un effort de transparence et de visibilité, ce qui émerge est plutôt l'opacité la plus complète autour du financement de ces nouvelles activités ${ }^{10}$. Le cas kenyan valide l'importance prise par les IAP dans la gestion des établissements d'enseignement supérieur. Par la norme ISO, de nouvelles procédures et normes bureaucratiques régulent, de manière inédite, l'UN. Allors que ce nouveau management public tend à combattre les lourdeurs bureaucratiques et ses gaspillages, on assiste à la formation d'une nouvelle bureaucratie qui se met en place par des normes (Hibou, 2012, 2013).

\subsection{Les effets contrastés des IAP dans le secteur de l'enseignement supérieur : l'exemple de l'UM}

Alors que la certification ISO 9001 doit être adoptée depuis plusieurs mois - le cas kenyan étant le parangon de la région - l'UM n'a pour le moment pas les revenus en interne pour certifier l'ensemble de ses activités. Comme le souligne un rapport récent: "The ISO certification was to be pursued in a phased approach beginning with identifying structures and processes such as laboratories and centres that are ready for certification. The underlying cause for the delay is the inadequate funding as more resources need to be committed to the assessment and certification process » (Makerere University, 2017, p. 57). Le principe de l'assurance qualité s'est, pour autant, déjà imposé à l'UM via l'ouverture de plusieurs bureaux d'assurance qualité sur ses campus, répondant ainsi aux recommandations générales de l'IUCEA et de ses partenaires allemands. Néanmoins, en Ouganda, les bureaux n'ont pas les ressources nécessaires pour fonctionner. Les principes de l'assurance qualité demeurent ainsi des labels fictifs, plutôt que des principes opérationnels ayant des conséquences décisives sur les pratiques des universitaires et l'organisation de l'institution. Comme le confirme une

\footnotetext{
${ }^{10}$ Etant donné que l'université génère la majorité de ses propres fonds comme une entreprise privée, les administrateurs déclarent qu'ils ne doivent pas divulguer publiquement ces chiffres en vue de la concurrence. D’après le résultat de nos recherches, concernant la première certification en 2008, l'Université de Nairobi a déboursé 42000 US\$. A ce montant doit se rajouter l'ensemble des formations du personnel (audits internes et externes) dans les différents départements. Ces services sont, par exemple, facturés 1000 US\$ par jour par l'« APEX Management Systems-Consultants LTD», l'un des organismes utilisé par l'Université de Nairobi. Entretien avec un responsable des formations aux certifications ISO réalisé le 15/11/2011, Nairobi.
} 
professeure et ancienne doyenne de la faculté des arts: "How can one talk of quality assurance ? Because even the department of the quality assurance itself, if you go there you will laugh! Two miserable people there, they cannot work ! How can they insure quality ? There is no quality assurance even in the quality assurance office itself ! $»{ }^{11}$. Le cas ougandais tend à nuancer l'impact des IAP sur le fonctionnement quotidien des établissements. Il nous rappelle surtout que la mise en œuvre d'un instrument suppose l'existence de moyens financiers et humains en l'occurrence - qui permet de le concrétiser.

Surtout, l'exemple de l'UM démontre comment les IAP peuvent se superposer à des enjeux locaux spécifiques et, après de nouvelles négociations et réappropriations par les acteurs, se voir détourner de leurs objectifs initiaux. Ce résultat provient de nos recherches sur le second instrument structurant le secteur de l'enseignement supérieur dans la région: le rôle des programmes de développement national dans le contenu des formations et le choix des filières des étudiants. Le cas de l'UM démontre en effet comment les acteurs locaux en charge des SHS se sont adaptés à ce nouveau contexte, qui visait pourtant à les mettre en péril. Pour le démontrer, on propose de partir de cette énigme : pourquoi les effectifs des filières en sciences sociales et humaines attirent encore le plus d'étudiants alors que, depuis les années 2000 et la promotion de ces documents cadres, la politique gouvernementale tend à modifier cet équilibre en faveur des sciences. En effet, même si l'on observe une hausse dans l'ensemble des filières scientifiques du nombre d'étudiants (respectivement 27, 21, 59 et $38 \%$ ), les étudiants inscrits en SHS restent encore très largement majoritaires (plus de 75\% des effectifs) entre 2007 et 2010, grâce à la hausse de 326\% des étudiants dans les humanités.

Tableau $\mathrm{n}^{\circ} 1$ : Effectif des étudiants par filière universitaire en Ouganda $(2007-2010)^{12}$

\begin{tabular}{|r|r|r|r|r|r|r|r|r|}
\hline & Sciences \\
sociales & Humanités & $\begin{array}{c}\text { Sciences } \\
\text { de } \\
\text { lagriculture }\end{array}$ & $\begin{array}{c}\text { Sciences } \\
\text { médicales }\end{array}$ & $\begin{array}{c}\text { Sciences } \\
\text { de } \\
\text { lingénieur }\end{array}$ & $\begin{array}{c}\text { Sciences } \\
\text { naturelles }\end{array}$ & Total & $\begin{array}{c}\text { Pourcentage } \\
\text { détudiants } \\
\text { ensciencessociales } \\
\text { ethumaines (\%) }\end{array}$ \\
\hline 2007 & 62901 & 3344 & 1678 & 3489 & 3447 & 8781 & 83640 & 79 \\
\hline 2008 & 58834 & 8033 & 1990 & 3971 & 3866 & 8928 & 85622 & 78 \\
\hline 2009 & 57279 & 12836 & 1627 & 4007 & 4001 & 10646 & 90396 & 76 \\
\hline 2010 & 60188 & 14259 & 2137 & 4230 & 5482 & 12137 & 98433 & 78 \\
\hline
\end{tabular}

Même si les études coûtent en moyenne plus chères en sciences qu'en sciences sociales ${ }^{13}$ facteur déterminant pour les étudiants dans le choix d'une filière - nous défendons que ce déséquilibre provient surtout des stratégies développées par les responsables des départements et des facultés en sciences sociales pour tirer profit de la mise en œuvre de ces IAP.

L'UM est la première institution de la région, depuis les Indépendances, a avoir engagé des réformes structurelles afin de répondre à l'importante restriction budgétaire du gouvernement

\footnotetext{
${ }^{11}$ Entretien réalisé le 23/11/2012, Kampala.

${ }^{12}$ Uganda National Council for Science and Technology, 2011, p. 31. Le tableau a été réalisé en collaboration avec Marie-Louise Penin du CNRS.

${ }^{13}$ Par exemple, en 2013-2014, les frais d'inscription pour les étudiants préparant une licence en étude de développement s'élèvent à 700,000 Ushs (soit environ 275 US\$) alors qu'en dentaire ils sont de 2,000,000 Ushs (soit environ 785 US\$).
} 
(Court, 1999 ; Kasozi, 2000, 2009 ; Musisi et Muwanga, 2003 ; Kwesiga et Ahikire, 2006 ; Mamdani, 2007 ; Provini, 2014, 2015, 2018). Face à ce contexte de crise, les facultés et les départements de SHS ont déployé des stratégies pour générer leurs propres revenus. Certains responsables ont décidé, au début des années 1990, de mettre en place de nouveaux cursus pour attirer des étudiants " privés ", c'est-à-dire des étudiants payant eux-mêmes leur frais de scolarité. À cette période, le pouvoir est très déconcentré au sein de l'institution. Chaque faculté dispose de la possibilité de mettre en place de nouveaux programmes sans que la décision finale ne soit validée par l'administration centrale. Les départements de SHS profite alors de la possibilité de pouvoir massifier plus facilement leurs cours par rapport aux unités en sciences, ces dernières devant effectuer un investissement financier plus important en termes de matériel et d'infrastructure. Le doyen de la faculté des arts initie ainsi un changement radical qui sera généralisée progressivement aux SHS, comme il nous l'explique : «cette faculté, dans les années 1990, était très démunie, elle était ridiculisée. Donc nous avons entrepris des réformes, et j'étais le doyen à l'époque, de 1992 à 2003. Donc nous avons beaucoup innové, avec les cours du soir, et la mise en place de différents cours qui n'existaient pas à l'époque [...]. Au moment où je l'ai quittée, plus du quart du revenu de l'université provenait de notre faculté. Et nous avions peut-être aussi le tiers des étudiants dans notre faculté. On ne pouvait pas rester avec le «bachelor [équivalent de la licence] of arts » qui n'attirait personne. Nous avons enrichi le «bachelor of arts » avec ce que nous avons appelé la professionnalisation, c'est-à-dire qu'on pouvait faire de l'histoire avec du management, que nous avons appelé "organisational studies". On pouvait faire de la littérature avec des études de secrétariat, pour avoir quelque chose à faire si on n'enseigne pas. On a créé de nouveaux programmes. L' " environmental management » était ici, l' " urban planning » était ici, le «tourism » était ici, les « development studies » étaient ici. Et quand les autres facultés se sont plaintes que ce n'était pas notre domaine, notre argument était de dire que "vous, vous ne les avez pas. Vous ne les avez pas créés donc laissez nous gérer $\gg{ }^{14}$.

De nouveaux cours, qui n'ont d'ailleurs pas toujours été accrédités officiellement (Mugerwa, 2012), ont donc vu le jour jusqu'à la fin des années 2000 : "environment management», " tourism management», "urban planning», " organisation studies », " communication skills », « mass communication », " social anthropology », " drama and dance », " tourism », " environment and secretarial studies », " development studies », et " development studies, human rights, ethics and human management» (Mamdani, 2007 : 65). Avec cette volonté de capter de nouveaux étudiants privés et donc générer des revenus inédits, la faculté des arts applique - bien avant la généralisation des programmes de développement national - le discours de la professionnalisation des cursus et de leur adaptabilité au marché de l'emploi. Il s'agit d'associer de nouveaux programmes, a priori plus séduisants, aux cours généralement enseignés. Par exemple, les étudiants peuvent étudier en même temps les études religieuses et la résolution de conflits, la géographie et le tourisme, les études linguistiques et le secrétariat, l'histoire et le développement ou encore la philosophie et le management public (Mamdani, 2007 : 53). La professionnalisation des cursus en SHS et l'adaptabilité des savoirs au marché de l'emploi, qui débutent au début des années 1990, sont légitimées par les plans de développement national des années 2000. Les doyens des facultés des SHS utilisent en effet les IAP pour défendre leurs pratiques et le développement de leurs facultés. Alors que les programmes de développement national doivent favoriser les cursus et les diplômes en science, les doyens des SHS les utilisent comme des outils pour légitimer leurs pratiques de professionnalisation des cursus. D'ailleurs, encore aujourd'hui, les stratégies des doyens des

\footnotetext{
${ }^{14}$ Entretien réalisé le 03/05/2012, Kampala.
} 
SHS ne consistent pas tant à s'aligner sur les recommandations du gouvernement pour recomposer les cursus et les savoirs que de générer des fonds et répondre à la demande de hausse de salaire des enseignants (Provini, 2018). Ces résultats remettent en cause l'ensemble des instruments construit depuis les années 2000 afin d'aligner la formation des élites ougandaises sur les besoins identifiés du marché. On comprend dès lors bien comment les administrateurs, qui reprennent à leurs comptes actuellement les programmes de développement national, engagent des effets inattendus et non-désirés initialement aux IAP.

\section{Conclusion}

Cette question de la mise en œuvre et des effets des IAP dans les universités kenyane et ougandaise nous permet de revenir sur les propositions de recherche de J.-P. Le Bourhis et P. Lascoumes qui soulignent l'importance de mettre en exergue les potentielles dynamiques de blocage, de conflits et d'oppositions des instruments. Ces entrées font encore partie des effets peu observés et analysés sur ces techniques de gouvernement (Le Bourhis, Lascoumes, 2014). Alors que l'étude de la fabrique des IAP au Kenya et en Ouganda présentent des résultats similaires - la nature des instruments est comparable et les acteurs promouvant leur développement sont analogues -, l'analyse de l'implémentation des instruments donnent à voir des résultats contrastés en Afrique de l'Est. Le cas kenyan valide plutôt l'importance prise par les IAP dans la gestion quotidienne des établissements d'enseignement supérieur : l'avènement de la norme ISO 9001 à l'UN est, par exemple, aujourd'hui l'un des parangons des procédures d'assurance qualité dans la région. L'exemple ougandais, en illustrant les effets originaux et non-désirés que peuvent avoir ces IAP lors de l'implémentation, tend à nuancer l'importance prise par ces instruments dans la régulation des universités estafricaines. Sur l'assurance qualité, l'UM n'a pas encore les moyens de la mettre réellement en œuvre. Surtout, concernant les programmes de développement national et leurs conséquences sur les savoirs, notre étude de cas met en exergue que les IAP sont l'objet de lutte de pouvoir et peuvent engendrer de nouvelles traductions. En s'insérant localement, ils sont agencés par des intérêts d'acteurs, des contextes historiques spécifiques et des pratiques bureaucratiques nécessairement singulières. L'exemple de l'UM nous rappelle que si l'ingénierie universitaire est le résultat d'une redéfinition de l'université dans les arènes internationales, régionales et nationales, elle peut être retraduite localement en fonction des acteurs universitaires. Ce résultat rejoint les travaux classiques des policy sciences sur l'implémentation des politiques publiques qui ont, très tôt (par exemple dans Pressman et Wildavsky, 1973), mis en lumière les décalages entre la fabrique et la mise en œuvre de l'action publique : " what can be called public policy, and thus has to be implemented, is the product of what has happened in the earlier stages of the policy process. Nevertheless, the content of that policy, and its impact on those affected, may be substantially modified, elaborated or even negated during the implementation stage » (Hill et Hupe, 2002: 6-7). Ce sont bien les acteurs « du bas» (Hassenteufel, 2011, p. 105-110), en l'occurrence les responsables administratifs des facultés de sciences sociales de l'UM, qui sont les principaux agents des IAP. Ils ont un rôle central dans la (non)concrétisation, les effets de traduction et de réadaptation locale de l'action publique, rejoignant là encore des travaux bien établis de la science politique (Lipsky, 1980 ; Sabatier, 1985, Dubois, 2010). Sur la base de ces premiers résultats, on pourrait même émettre l'hypothèse que les décalages entre la fabrique et la mise en œuvre de l'action publique, notamment des IAP, seraient plus importants en Afrique qu'au Nord. Peut-être plus qu'ailleurs, la configuration des politiques publiques en Afrique semble être caractérisée par ces décalages spatial et temporel entre la fabrique et l'implémentation de l'action publique, où s'agrègent et se greffent de nouveaux acteurs, enjeux politiques, administratifs et relations de pouvoir lors de la phase d'implémentation, réorientant l'action négociée antérieurement (voir 
les résultats du programme de recherche FAPPA ${ }^{15}$ ). Alors qu'on observe une faible participation d'acteurs « du bas » et de la « société civile » dans les processus de fabrique des politiques publiques en Afrique - séquence régulée par les acteurs internationaux et nationaux et qui s'explique par une faible différenciation de l'Etat et de la société (Darbon, Provini, 2018) -, la participation d'acteurs «du bas » serait d'autant plus significative au moment de la mise en œuvre (par exemple au Kenya et en Tanzanie, voir Provini et Schlimmer 2019 a, 2019b).

Ces deux études de cas tendent également à apporter des résultats stimulants pour les travaux sur les réformes de l'enseignement supérieur en Afrique. Le cas kenyan confirme l'importance des instruments construits par les acteurs internationaux, régionaux et nationaux, validant l'hypothèse de politiques publiques d'enseignement supérieur en partie externalisées. On observe bien de nouveaux instruments qui organisent désormais les activités administratives et académiques des établissements publics comme privés. Les procédures et les normes bureaucratiques, encouragées par une vision entrepreneuriale des établissements, engagent une nouvelle conceptualisation du rôle et des missions de l'université publique. Le savoir et savoir-faire hérités du secteur privé - qui se donnent à voir par la multiplication des rapports, des tableaux, des organigrammes, des graphiques, des chiffres, des indicateurs, des objectifs et des buts organisationnels - planifient et implémentent les contours d'une nouvelle définition du paysage de l'enseignement supérieur que le Kenya illustre de manière saillante. De la même manière qu'au Nord, le secteur de l'enseignement supérieur est régulé par un nouveau paradigme qui organise le management des universités publiques comme privées. Néanmoins, le cas ougandais invite à nuancer l'importance prise par ces nouvelles normes sur l'assurance qualité et sur les savoirs. Il met plutôt l'accent sur les faibles capacités de l'établissement pour implémenter les procédures d'assurance qualité et sur les stratégies des acteurs « du bas » pour s'accommoder et tirer profit du changement. Plus largement, cette étude de cas de l'UM nous rappelle l'importance des processus de réappropriation et d'hybridation des politiques publiques universitaires. Même dans un Etat et un secteur de l'enseignement supérieur qui dépendent fortement de l'aide internationale, de l'intervention des bailleurs de fonds et des partenariats étrangers, l'action publique semble demeurer un processus dynamique de coproduction, ne serait-ce que dans la phase de la mise en œuvre. Ce résultat rejoint les travaux sur d'autres secteurs sur le continent (Lavigne Delville, 2010 ; Provini, Schlimmer, 2016) et nous invite à multiplier les recherches sur l'implémentation de l'action publique sur le continent qui demeure encore lacunaire.

\section{Bibliographie}

Akkari, A. \& Payet, J.-P. (Eds.) (2010). Transformations des systèmes éducatifs dans les pays du Sud. Entre globalisation et diversification. Bruxelles: De Boeck.

Bayart, J.-F. (2012). Sortir du national-libéralisme. Croquis politiques des années 2004-2012. Paris: Karthala.

Brock-Utne, B. (2003). Formulating Higher Education Policies in Africa : The Pressure from External Forces and the Neoliberal Agenda. JHEA/RESA, 1(1), 24-56.

\footnotetext{
${ }^{15}$ Le programme de recherche FAPPA (Faire des politiques publiques en Afrique), porté à Sciences Po Bordeaux, consiste à étudier et à comparer les processus de négociation, de formulation et de mise en œuvre des politiques publiques dans six pays (Maroc, Madagascar, Côte d'Ivoire, Mozambique, Kenya et Tanzanie) et quatre secteurs (gestion des ressources naturelles, politiques de la ville, réformes de l'éducation, politiques sociales). Voir https://fappa.hypotheses.org/.
} 
Bruno, I. (2008). La recherche scientifique au crible du benchmarking. Petite histoire d'une technologie de gouvernement. Revue d'histoire moderne \& contemporaine, 55(4 bis), 28-45.

Bugwabari, N., Cazenave-Piarrot, A., Provini, O. \& Thibon, C. (Eds.) (2012). Universités, universitaires en Afrique de l'Est. Nairobi/Paris: Institut français de recherche en Afrique/Karthala.

Charlier, J.-E. (2006). Savants et sorciers. Les universités africaines francophones face à la prétendue universalité des critères de qualité. Education et Sociétés, 2(18), 93-108.

Charlier, J.-E. \& Croché, S. (2010). L'inéluctable ajustement des universités africaines au processus de Bologne. Revue française de pédagogie, 172, 77-84.

Charlier, J.-E. \& Croché, S. (2012). L'influence normative du processus de Bologne sur les universités africaines francophones. Education et Sociétés, 29, 87-102.

Charton, H. \& Owuor, S. (2008). De l'intellectuel à l'expert. Les sciences sociales africaines dans la tourmente : le cas du Kenya. Revue internationale d'éducation Sèvres, 49, 107-119.

Coloma, T. (2013). Des avenues pour la corruption. Le Monde diplomatique, avril, 13.

Commission for Higher Education Kenya, Tanzania Commission for Universities \& National Council for Higher Education Uganda (2009). Credit Accumulation and Transfer System, East Africa. Minimum Standards for Courses of Study in Basic Sciences Undergraduate Programmes. Kampala: Commission for higher education Kenya/Tanzania commission for universities/National council for higher education Uganda.

Court, D. (1999). Financing Higher Education in Africa: Makerere, the Quiet Revolution. Working paper: Banque mondiale/Fondation Rockefeller.

Darbon, D. \& Provini, O. (2018). Penser l'action publique en contextes africains : les enjeux d'une décentration. Gouvernement et action publique, 7(2), 9-29.

Dardot, P. \& Laval, C. (2009). La nouvelle raison du monde. Essai sur la société néolibérale. Paris: La Découverte.

Dubois, V. (2010). Politiques au guichet, politiques du guichet. In O. Borraz \& V. Guiraudon (Dir.), Politiques publiques, 2. Changer la société (pp.265-286). Paris: Presses de Sciences Po.

Fourie, E. (2014). Model Students: Policy Emulation, Modernization, and Kenya's Vision 2030. African Affairs, 113(453), 540-562.

Government of the Republic of Kenya (2007). Kenya Vision 2030. A Globally Competitive and Prosperous Kenya. Nairobi: Government of the Republic of Kenya.

Government of the Republic of Kenya (2012). Performance Contracting Guidelines on the Vision 2030 Project Indicators. Nairobi: Government of the Republic of Kenya. 
Halpern, C., Lascoumes, P. \& Le Galès, P. (Dir.) (2014). L'instrumentation de l'action publique. Paris: Presses de Sciences Po.

Hansert, C., Kuria, M. \& Nkunya, M. H. H. (2012). Joint Capacity Development for Regional Quality Assurance: the IUCEA-DAAD-HRK East African Quality Assurance Initiative. Working paper: ADEA.

Hassenteufel, P. (2011). Sociologie politique : l'action publique. Paris: Armand Colin $\left(2^{\text {ème }}\right.$ édition).

Hibou, B. (2012). La bureaucratisation du monde à l'ère néolibérale. Paris: La Découverte.

Hibou, B. (Dir.) (2013). La bureaucratisation néolibérale. Paris: La Découverte.

Hill, M. \& Hupe, P. (2002). Implementing Public Policy. Thousand Oaks: Sage.

Kasozi, A. B. K. (2000). University Education in Uganda. Challenges and Opportunities for Reform. Kampala: Fountain.

Kasozi, A. B. K. (2009). Financing Uganda's Public Universities. An obstacle to Serving the Public Good. Kampala: Fountain Publishers.

Kithinji, M. M. (2012). An Imperial Enterprise : the Making and Breaking of the University of East Africa, 1949-1969. Canadian Journal of African Studies, 46(2), 195-214.

Knight, J. \& Woldegiorgis, E. T. (Eds.) (2017). Regionalization of African Higher Education. Progress and Prospects. Rotterdam: Sense Publishers.

Kwesiga, J. C. \& Ahikire, J. (2006). On Student Access and Equity in a Reforming University : Makerere in the 1990s and Beyond. JHEA/RESA, 4(2), 1-46.

Lange, M.-F. \& Henaff, N. (2015). Politiques, acteurs et systèmes éducatifs entre internationalisation et mondialisation. Introduction. Revue Tiers Monde, 223, 11-28.

Lascoumes, P. \& Le Galès, P. (2004). Gouverner par les instruments, Paris: Presses de Sciences Po.

Lascoumes, P. \& Le Galès, P. (2010). Instrument. In L. Boussaguet, S. Jacquot \& P. Ravinet (Dir.), Dictionnaire des politiques publiques (pp.325-335). Paris: Presses de Sciences Po.

Lascoumes, P. \& Simard, L. (2011). L'action publique au prisme de ses instruments. Revue française de science politique, 61(1), 5-22.

Lavigne Delville, P. (2010). La réforme foncière rurale au Bénin. Emergence et mise en question d'une politique instituante dans un pays sous régime d'aide. Revue française de science politique, 60 (3), 467-491.

Lebeau, Y. (2003). Classement et déclassement des universités au Nigeria. De l'uniformité des procédures d'admission à la multiplicité des stratégies de contournement. Cahiers d'études africaines, 1-2(169-170), 209-233. 
Le Bourhis, J.-P. \& Lascoumes, P. (2014). En guise de conclusion: Les résistances aux instruments de gouvernement. Essai d'inventaire et de typologie des pratiques. In C. Halpern, P. Lascoumes \& P. Le Galès (Dir.). L'instrumentation de l'action publique (pp.493-520). Paris: Presses de Sciences Po.

Leclerc-Olive, M., Scarfo Ghellab, G. \& Wagner, A.-C. (Dir.) (2011). Les mondes universitaires face au marché. Circulation des savoirs et pratiques des acteurs. Paris: Karthala.

Léon, A. (2004). L'intégration Est-africaine. L'East African Community: vers une intégration régionale des espaces. Les Cahiers d'Afrique de l'Est / The East African Review, 23.

Lipsky, M. (1980). Street-Level Bureaucracy: Dilemmas of the Individual in Public Services, New York: Russel Sage Foundation.

Makerere University (2007). Strategic Plan 2007/08-2017/18. Planning and Development Department. Kampala: Makerere University.

Makerere University (2008). Strategic Plan 2008/09-2018/19. Kampala: Makerere University.

Makerere University (2017). Strategic Plan Review Report. Kampala: Makerere University.

Mamdani, M. (2007). Scholars in the Marketplace: The Dilemmas of Neo-Liberal Reform at Makerere University (1989-2005). Saint-Paul: Codesria.

Materu, P. (2007). Higher Education Quality Assurance in Sub-Saharan Africa. Status, Challenges, Opportunities, and Promising Practices. World Bank Working Paper, 124.

Mngomezulu, B. R. (2004). A Political History of Higher Education in East Africa. The Rise and Fall of the University of East Africa, 1937-1970. Houston: UMI.

Mugerwa, Y. (2012). 33\% of Mak Courses Are Illegal, AG Says. Daily Monitor, 9 avril, 3.

Munene, I. I. (2013). «... University Is ISO 9000:2008 Certified»: Neoliberal Echoes, Knowledge Production and Quality Assurance in Kenyan State Universities. JHEA/RESA, 11 (1-2), 161-182.

Musisi, N. B. \& Muwanga, N. K. (2003). Makerere University in Transition 1993-2000: Opportunities and Challenges. Oxford/Kampala: James Currey/Fountain Publishers.

Pressman, J. L. \& Wildavsky, A. B. (1973), Implementation. Berkeley: University of California Press.

Provini, O. (2012). Croquis du nouveau management public dans l'espace universitaire estafricain. Mambo!, 10(6). 
Provini, O. (2014). The Political Economy of Makerere Reforms via a Qualitative Approach. An Interview with the Professor Ruth Mukama. Les Cahiers d'Afrique de l'Est / The East African Review, 49, 143-160.

Provini, O. (2015). La circulation des réformes universitaires en Afrique de l'Est. Les politiques de l'enseignement supérieur au regard de la sociologie de l'action publique et de l'Etat. Thèse de Doctorat: Université de Pau et des Pays de l'Adour.

Provini, O. (2016). La circulation des réformes universitaires en Afrique de l'Est. Logiques de convergences et tendances aux divergences. Cahiers de la recherche sur l'éducation et les savoirs, 15 .

Provini, O. (2018). Quand les doyens et les enseignants lâchent leurs étudiants : une nouvelle lecture des réformes à l'Université de Makerere en Ouganda (1986-2000). Gouvernement et action publique, 7(2), 117-139.

Provini, O. (2019). La mise en marché de l'enseignement supérieur au Kenya. Critique internationale, 4(85), 145-164

Provini, O. \& Schlimmer, S. (2016). Négocier l'action publique dans un Etat sous régime d'aide: une analyse comparée des politiques de l'enseignement supérieur et du foncier en Tanzanie. Revue internationale de politique comparée, 23(2), 199-223.

Provini, O. \& Schlimmer, S. (2019a). Etude de cas: Kenya, Livrable FAPPA : Etudes sectorielles, working paper, mai

Provini, O. \& Schlimmer, S. (2019b). Etude de cas : Tanzanie. Livrable FAPPA : Etudes sectorielles, working paper, mai

Sabatier, P. (1985). Top-Down and Bottom-Up Approaches to Implementation Research: a Critical Analysis and Suggested Synthesis. Journal of Public Policy, 6(1), 21-48.

Samuel, B. (2017). Planifier en Afrique. Politique africaine, 1(145), 5-26.

Shabani, J., Okebukola, P. \& Oyewole, O. (2017). Regionalization of Quality Assurance in Africa. In J. Knight \& E. T. Woldegiorgis (Eds.). Regionalization of African Higher Education. Progress and Prospects (pp.93-112). Rotterdam: Sense Publishers.

Speich, D. C. (2017). Les statistiques comme mode de communication politique. Le cas des premiers plans de développement au Kenya. Politique africaine, 1(145), 85-108.

Tade, A. A. (2012). Beyond Reforms: The Politics of Higher Education Transformation in Africa. African Studies Review, 53(1), 21-40.

The Inter-University Council for East Africa (2010a). A Road Map to Quality. Handbook for Quality Assurance in Higher Education. Volume 1: Guidelines for Self Assessment at Program Level. Kampala: IUCEA/DAAD/German Rector's Conference. 
The Inter-University Council for East Africa (2010b). A Road Map to Quality. Handbook for Quality Assurance in Higher Education. Volume 2: Guidelines for External Assessment at Program Level. Kampala: IUCEA/DAAD/German Rector's Conference.

The Inter-University Council for East Africa \& East African Community (2009). Study on the Harmonization of the East African Education Systems. Arusha: IUCEA/EAC.

The Republic of Uganda (1998). Vision 2025. Kampala: The Republic of Uganda.

The Republic of Uganda (2006). Vision 2035. Kampala: The Republic of Uganda.

The Republic of Uganda (2013). Vision 2040 Draft. Kampala: The Republic of Uganda.

Uganda National Council for Science and Technology (2011). Science, Technology and Innovation in Uganda. Status Report 2009/2010. Kampala: Uganda National Council for Science and Technology.

Uwakwe, C. B. U., Falaye, A. O., Emunemu, B. O. \& Adelore, O. (2008). Impact of Decentralization and Privatization on the Quality of Education in Sub-Saharan Africa: The Nigerian Experience. European Journal of Social Sciences, 7(1), 160-170.

Zeleza, P. T. \& Olukosho, A. (Eds.) (2004). African Universities in the Twenty-First Century. Volume I: Liberalisation and Internationalisation. Dakar: CODESRIA. 